\title{
AN INTRODUCTION TO THE CLASSIFICATION OF THE STUDY DESIGNS IN CLINICAL TRIALS
}

\author{
A.R. Muralidharn \\ Assistant Professor of Biostatistics, Post Graduate department of Biostatistics, \\ College of Natural and computational sciences, Debre Berhan University, Debre Berhan, Ethiopia.
}

\begin{abstract}
Day by day there is a high volume of clinical trials designed and developed; even there are some gaps and doubts to develop these designs. This article will provide an Idea in classifying such designs and also put effort on knowledge about these designs. The author has this idea from several questions arise by students and trainers. Here author want to acknowledge such groups. In this article a discussion made on several study designs in clinical trials which are more frequently used and even some of the designs may omitted based on its importance of the study search.
\end{abstract}

Keywords: clinical trials, clinical design, observational studies, experimental studies, clinical research, pyramid of clinical design, evidence-based medicine

\section{INTRODUCTION}

Clinical trial is an emerging field for the current topic of agenda for all countries. By clinical trial we ensure the two criteria's to the society, they are Efficacy and Safety. Because the trials in the clinical environment will be based on human subjects and the innovative results will be going to serve for the society of people to recover or heal or improve their quality of life and longevity of their life. Hence it is important to aware the concepts, designs, trial and its application for those involve in such trials.

Clinical trials are research studies performed in Human subjects that are aimed at evaluating a medical, surgical, device or behavioral intervention. They are the primary way that clinician find out if a new treatment, like a new drug or diet or medical device is safe and effective in subjects. Often a clinical trial is used to learn if a new treatment is more effective and/or has less harmful side effects than the standard treatment with ensuring of safety and efficacy.
There are some common terms can replace the word "Clinical Trials" as Medical research, clinical research, bio statistical research and so on. In any clinical trial, the ultimate goal is to evaluate the effect of test treatment as compared to a control (Placebo, standard therapy or an active control agent). To ensure this trial in a success way, a well-designed study protocol is essential. A protocol is a Blue print or Path plan document. Protocol will explain the details how a clinical trial is to be carried out and how the data are to be collected and analyzed.

Since clinical trials are ensuring the quality and integrity of the clinical investigational process in terms of Planning, executing, conducting, and the interpretation made on the data of clinical trials. Finally, it is more important that any clinical trial must approved by Food and Drug Administration (FDA), USA. According to FDA, there is at least two adequate and well-controlled clinical trials be conducted to provide substantial evidence regarding the effectiveness of the drug product under investigation (FDA, 1988). According to the FDA 1988 guideline for Format and Content of the Clinical and Statistical Sections of New Drug Applications, an adequate and well-controlled study is defined as a study that meets the characteristics of the following: (i) objectives, (ii) methods of analysis, (iii) design, (iv) selection of subjects, (v) assignment of subjects, (vi) participants of studies, (vii) assessment of responses, and (viii) assessment of effect. In the study protocol, it is essential to clearly state the study objectives of the study, specific objectives, and hypothesis.

The study design must be valid in order to provide. a fair and unbiased assessment of the treatment effect as compared to a control. Target patient population should be defined through the inclusion/exclusion criteria to assure the disease conditions under study. 
Thus it is necessary to get knowledge about the study design and its importance. To enrich the knowledge of study design which are mostly used in clinical trials, this article will give some basic knowledge about such designs.

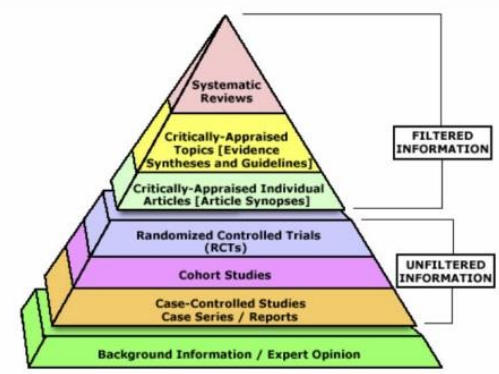

Figure 1: The Pyramid of clinical trials

\section{Classification of the study designs in Clinical trials}

The pyramid of clinical trials in Figure 1, explicitly shows the ordered form of the clinical trial design. There are several different schemes for classifying study designs. We have adopted one that divides studies into those in which the subjects were merely observed, sometimes called observational studies (Figure 3), and those in which some intervention was performed, generally called experiments or experimental studies. (Figure 4). This approach is simple and reflects the sequence an investigation sometimes takes. Figure 2 tries to express the classification of the designs.

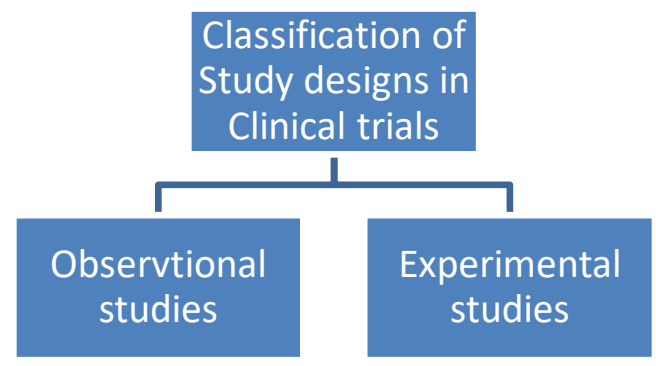

Figure 2: Classification of study design in Clinical trials

Observational studies involve no intervention - an observational system without any procedure or treatment. Experimental studies involve an intervention - an investigator-controlled maneuver, such as a drug, a procedure, or a treatment - and interest lies in the effect the intervention has on study subjects. Of course, both observational and experimental studies may involve animals or objects, but most studies in medicine involve people.

In Figure 3, the classification of design involved with Observational studies are presented and these trials are discussed elaborately

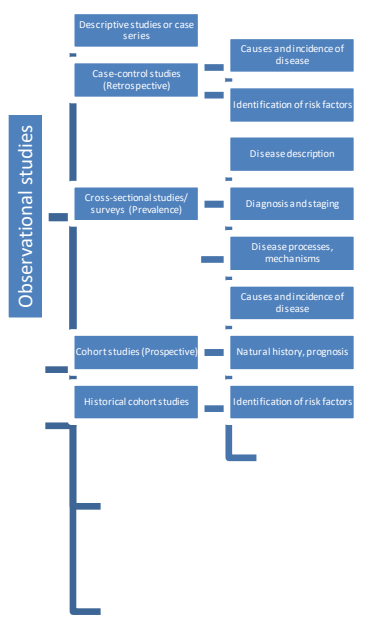

Figure 3: Observational study designs in Clinical trial

\section{OBSERVATIONAL STUDIES}

Observational studies are an important category of study designs. Well-designed observational studies have been shown to provide results similar to Randomized controlled trials(RCT). challenging the belief that observational studies are a second -rate. Observational studies fall under the category of analytic study designs and further observational studies are of four sub- types: case-series, casecontrol, cross-sectional (including surveys), and cohort studies. When certain characteristics of a group (or series) of patients (or cases) are described in a published report, the result is called a case-series study; it is the simplest design in which the author describes some interesting or intriguing observations that occurred for a small number of patients. A caseseries report is a simple descriptive account of interesting characteristics observed in a group of patients. Case-series studies frequently lead to the generation of hypotheses that are subsequently investigated in a case-control, cross-sectional, or cohort study. These three types of studies are defined by the period of time the study covers and by the direction or focus of the research question.

Cohort and case- control studies generally involve an extended period of time defined by the point when the study begins and the point when it ends; some process occurs, and a certain amount of time is 
required to assess it. For this reason, both cohort and case- control studies are sometimes also called longitudinal studies. The major difference between them is the direction of the inquiry or the focus of the research question: Cohort studies are forward looking, from a risk factor to an outcome, whereas case-control studies are backward-looking, from an outcome to risk factors. The cross-sectional study analyzes data collected on a group of subjects at one time. Kleinbaum and colleagues (1997) describe a number of hybrids or combinations of these designs.

\section{Descriptive or Case-series}

A case series presents several similar cases-is to make observations about patients with defined clinical characteristics. A case-series report is a simple descriptive account of interesting characteristics observed in a group of patients. Caseseries reports generally involve patients seen over a relatively less time. In general, the case-series studies do not include control subjects, persons who do not have the disease or condition being described. Some investigators would not include case-series in a list of types of studies because they are generally not planned studies and so not involve any research hypothesis. It is noted to be noted that, case-series studies because of their important descriptive role as a precursor to other studies.

The design is a simple description of the clinical data, preferably from a very well-defined group of individuals, without reference to a comparison group. Observations in these reports should be comprehensive and detailed enough to permit recognition of similar cases by the reader. The report should include a clear definition of the phenomenon under study.

Findings are usually presented as needed to illustrate the phenomenon, such as frequency of a given "discrete" (i.e., present/absent) variable or mean or median of a continuous variable (e.g., age or blood pressure) in the study series. Important subgroups, such as those defined by sex or age, may need stratified data presentation. In a case series, analysis is limited to descriptive variables such as proportions or means with standard errors. Interpretations and conclusions should include a summary of the new phenomenon illustrated in the report, reference to previous, related observations, and suggestions of etiology or of further studies needed. An important question is whether the described series is representative$$
\text { of }
$$

patients with the disorder such that conclusions can be generalized. This is often difficult to determine in initial case reports and case series, and it may well call for other investigators to identify and describe similar cases.

\section{Case-control studies}

Patients with a certain specific outcome or disease and an appropriate group of controls without the outcome or disease are selected and then information is obtained on whether the subjects have been exposed to the factor under investigation. There should be a serious consideration of appropriate choice of controls, matching and so on.

Case - control studies begin with the absence or presence of an outcome and then look backward in time to try to detect possible causes or risk factors that may have been suggested in a case-series report. The cases in case-control studies are individuals selected on the basis of some disease or outcome; the controls are individuals without the disease or outcome.

The history or previous events of both cases and controls are analyzed in an attempt to identify a characteristic or risk factor present in the cases' histories but not in the controls' histories
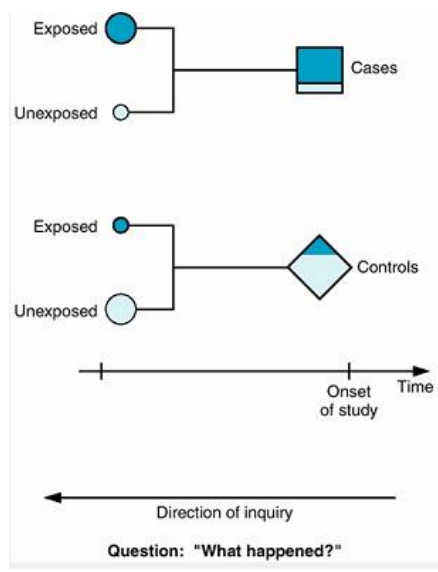

Figure 4: Schematic diagram of case-control study design

Source: Encyclopedia of Statistical Sciences, Vol 8. Wiley, 1988.

Figure 4, represent the schematic diagram of casecontrol study design. That subject in the study are chosen at the onset of the study after they are known to be either cases with the disease or outcome (squares) or controls without the disease or outcome (diamonds). The histories of cases and controls are 
examined over a previous period to detect the presence (shaded areas) or absence (unshaded areas) of predisposing characteristics or risk factors, or, if the disease is infectious, whether the subject has been exposed to the presumed infectious agent.

In the case of case-control designs, the nature of the inquiry is backward in time, as indicated by the arrows pointing backward as the figure mentioned direction of inquiry is retrospective, nature of the research process in clinical research design. As characterize case-control studies as studies as studies that ask "What happened?" In fact, they are sometimes called retrospective studies because of the direction of inquiry. Case-control studies are longitudinal as well, because the inquiry.

These case-control studies are longitudinal as well, because the inquiry covers a period of time. According to Olsen et.al (2003) they compared patients who had a surgical infection spinal fusion with patients who developed no infection (casecontrol), it was found that length of hospital stay and readmission rates were greater with patients with infections. A postoperative incontinence was one of the risk factors associated with the development of infection.

Investigators sometimes use matching to associate controls with cases on characteristics such as age and sex. If an investigator feels that such characteristics are so important that an imbalance between the two groups of patients would affect any conclusions, the patients or subjects employ matching. This process ensures that both groups will be similar with respect to important characteristics that may otherwise could or confound the conclusions.

Deciding whether a published study is a case-control study or a case-series report is not always easy. Confusion arises because both types of studies are generally conceived and written after at the fact rather than having been planned. The easiest way to differentiate between them is to ask whether the author's purpose was to describe a phenomenon or to attempt to explain it by evaluating previous events. If the purpose is simple description, chances are the study is a case-series report.

\section{Cross-Sectional Studies}

The third type of observational study goes by all of the following names: cross-sectional studies, surveys, epidemiologic studies, and prevalence studies. We use the term "cross-sectional" because it is descriptive of the time line and does not have the connotation that the terms "surveys" and "prevalence" do. Cross-sectional studies analyze data collected on a group of subjects at one time rather than over a period of time. Cross-sectional studies are designed to determine "What is happening?" right now. Subjects are selected and information is obtained in a short period of time (Figure 2-2; note the short time line). Because they focus on a point in time, they are sometimes also called prevalence studies. Surveys and polls are generally crosssectional studies, although surveys can be part of a cohort or case-control study. Cross-sectional studies may be designed to address research questions raised by a case-series, or they may be done without a previous descriptive study.

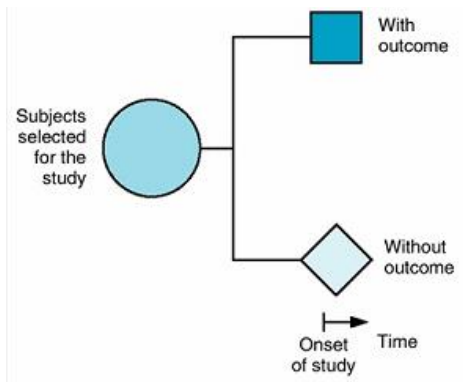

No direction of inquin

Question: "What is happening?"

Figure 5: Schematic diagram of cross-sectional study design

Source: Encyclopedia of Statistical Sciences, Vol 8. Wiley, 1988.

A study that examines the relationship between diseases or other health-related characteristics and other variables of interest as they exist in a defined population at one particular time (ie exposure and outcomes are both measured at the same time). For quantifying the prevalence of risk factor and for quantifying the accuracy of a diagnostic test this study will be the best.

\section{Surveys}

Surveys are especially useful when the goal is to gain insight into a perplexing topic or to learn how people think and feel about an issue. Surveys are generally cross-sectional in design, but they can be used in case-control and cohort studies as well. Caiola and Litaker (2000) wanted to know the factors that influence fellows to select a specific general internal residency fellowship program. Because they did not know the names and addresses of the fellows, the authors sent a questionnaire to the program directors and asked them to distribute the 
questionnaires to the fellows. We examine this study in more detail in Chapter 11 and illustrate how the authors asked the questions on the survey. Many times investigators use preexisting surveys rather than creating their own, especially if good questionnaires already exist. Patenaude and colleagues (2003) asked medical students at a Canadian medical school to complete a questionnaire on moral reasoning (the Kohlberg Moral Judgment Interview). They wanted to learn how moral reasoning progressed over time, so they gave the questionnaire at the beginning of medical school and again at the end of the third year. They learned that the stage of moral development did not change in about $70 \%$ of the students, whereas it either decreased or increased in $15 \%$. The authors had expected the level of moral reasoning to increase, and the results of the study prompted them to raise questions about the possible features of medical education that might inhibit its development. Interviews are sometimes used in surveys, especially when it is important to probe reasonsor explanations more deeply than is possible with a written questionnaire. Kendler and colleagues (2003) wanted to investigate the role of genetic and environmental risk factors for substance abuse. They studied six classes of illicit substances to learn whether substance use disorders are substance-specific. After interviewing almost 1200 sets of adult male twins, they concluded that environmental experiences unique to a given individual are primarily responsible for whether the person misuses one class of psychoactive

substances over another. Increasingly, surveys are performed using existing databases of information. As an illustration, Huang and Stafford (2002) used survey data from the National Ambulatory M edical Care Survey to examine the relationship between demographics and clinical characteristics of women who visit primary care physicians and specialists for urinary tract infection. Using preexisting databases can have a number of advantages, such as saving time and effort, but many national surveys use complicated designs; and it is important to know what these are.

Many countries and states collect data on a variety of conditions to develop tumor registries and databases of cases of infectious disease. Diermayer and colleagues (1 999), a presenting problem in Chapter 4, analyzed epidemiologic surveillance data from the State of Oregon and reported an increase in the overall incidence rate of meningococcal disease from 2 cases/1 00,000 population during 1987-1 992 to 4 . 5 cases/1 00,000 in 1 994. Epidemiologists from
Oregon and the Centers for Disease Control in Atlanta, Georgia, wanted to know if the increased number of cases of meningococcal disease indicated a transition from endemic to epidemic disease. They also sought these other features of an epidemic: the predominance of a single bacterial strain rather than a heterogeneous mix of strains and a shift in age distribution of cases toward older age groups.

\section{Cohort Studies}

A cohort is a group of people who have something in common and who remain part of a group over an extended time. In medicine, the subjects in cohort studies are selected by some defining characteristic (or characteristics) suspected of being a precursor to or risk factor for a disease or health effect. Cohort studies ask the question "What will happen?" and thus, the direction in cohort studies is forward in time. Figure 2-3 illustrates the study design. Researchers select subjects at the onset of the study and then determine whether they have the risk factor or have been exposed. All subjects are followed over a certain period to observe the effect of the risk factor or exposure. Because the events of interest transpire after the study is begun, these studies are sometimes called prospective studies

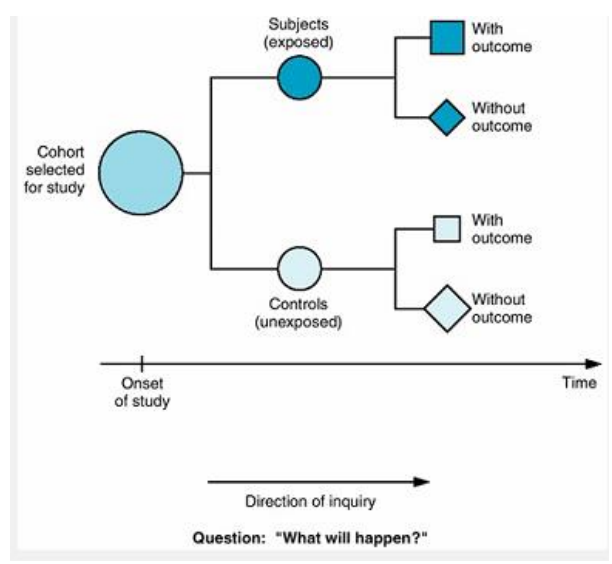

Figure 6: Schematic diagram of a cohort study design

\section{Typical Cohort Studies}

A classical cohort study with which most of you are probably familiar is the Framingham study of cardiovascular disease. This study was begun in 1 948 to investigate factors associated with the development of atherosclerotic and hypertensive cardiovascular disease, for which Gordon and Kannel (1 970) reported a comprehensive 20-year follow-up. 


\section{International Journal of Engineering Applied Sciences and Technology, 2020 \\ Vol. 4, Issue 11, ISSN No. 2455-2143, Pages 498-508 \\ Published Online March 2020 in IJEAST (http://www.ijeast.com)}

More than 6000 citizens in Framingham, Massachusetts, agreed to participate in this longterm study that involved follow-up interviews and physical examinations every 2 years. Many journal articles have been written about this cohort, and some of the children of the original subjects are now being followed as well.

Cohort studies often examine what happens to the disease over time - the natural history of the disease. $\mathrm{M}$ any studies have been based on the Framingham cohort; hundreds of journal articles are indexed by $\mathrm{M}$ EDLIN E. M any studies deal with cardiovascular related conditions for which the study was designed, such as blood pressure and pulse pressure as predictors of congestive heart failure $(\mathrm{H}$ aider et al, 2003), but this very rich source of data is being used to study many other conditions as well. For instance, two recent articles examined the life expectancy of adults who are obese (Peeters et al, 2003) and the relation of bone mass to development of prostate cancer (Zhang et al, 2002).

Although the Framingham Heart Study is very long term, many cohort studies follow subjects for a much shorter period. A presenting problem in Chapters 5 describes a cohort study to determine the effect of cholecystectomy on bowel habits and bile acid absorption (Sauter et al, 2002). Fifty-one patients undergoing cholecystectomy were evaluated before, 1 month after, and 3 months after surgery to detect changes such as abdominal pain, flatulence, and dyspepsia.

\section{Historical Cohort Studies}

Many cohort studies are prospective; that is, they begin at a specific time, the presence or absence of the risk factor is determined, and then information about the outcome of interest is collected at some future time, as in the two studies described earlier. One can also undertake a cohort study by using information collected in the past and kept in records or files.

For example, Shipley and his coinvestigators (1 999) wanted to assess study outcomes in men with prostate cancer treated with a specific type of radiation therapy (see Chapter 4). Six medical centers had consistently followed a group of patients who had previously been treated with this therapy. Shipley used existing records to look at survival and tumor recurrence in 1607 men who were treated between 1 988 and 1995 and had had at least four prostatespecific antigen measurements after radiation. This approach to a study is possible if the records on follow-up are complete and adequately detailed and if the investigators can ascertain the current status of the patients.

Some investigators call this type of study a historical cohort study or retrospective cohort study because historical information is used; that is, the events being evaluated actually occurred before the onset of the study (Figure 2-4). Note that the direction of the inquiry is still forward in time, from a possible cause or risk factor to an outcome. Studies that merely describe an investigator's experience with a group of patients and attempt to identify features associated with a good or bad outcome fall into this category, and many such studies are published in the medical literature.

The time relationship among the different observation study designs is illustrated in Figure 2-5. The figure shows the timing of surveys, which have no direction of inquiry, case-control designs, which look backward in time, and cohort studies, which look forward in time.

\section{Comparison of Case-Control and Cohort Studies}

Both case-control and cohort studies evaluate risks and causes of disease, and the design an investigator selects depends in part on the research question. $\mathrm{H}$ enderson and colleagues (1 997) undertook a cohort study to look at the risk factors for depression in the elderly. After an initial interview to collect information on potential risk factors, the investigators reinterviewed the subjects 3-6 years later to reassess their status. The investigators could have designed a case-control study had they asked the research question as: "Among elderly people exhibiting dementia or cognitive decline, what are the likely precursors or risk factors?" They would need to ascertain the patients' mental status in the past and any other potential reasons that might be associated with their present condition. As this illustration shows, a cohort study starts with a risk factor or exposure and looks at consequences; a case-control study takes the outcome as the starting point of the inquiry and looks for precursors or risk factors. Generally speaking, results from a well-designed cohort study carry more weight in understanding a disease than do results from a case-control study. A large number of possible biasing factors can play a role in case-control studies, and several of them are discussed at greater length in Chapter 13 . In spite of their shortcomings with respect to establishing causality, case-control studies are frequently used in medicine and can provide useful 


\section{International Journal of Engineering Applied Sciences and Technology, 2020 Vol. 4, Issue 11, ISSN No. 2455-2143, Pages 498-508 \\ Published Online March 2020 in IJEAST (http://www.ijeast.com)}

insights if well designed.

They can be completed in a much shorter time than cohort studies and are correspondingly less expensive to undertake. Case-control studies are especially useful for studying rare conditions or diseases that may not manifest themselves for many years. In addition, they are valuable for testing an original premise; if the results of the case-control study are promising, the investigator can design and undertake a more involved cohort study.

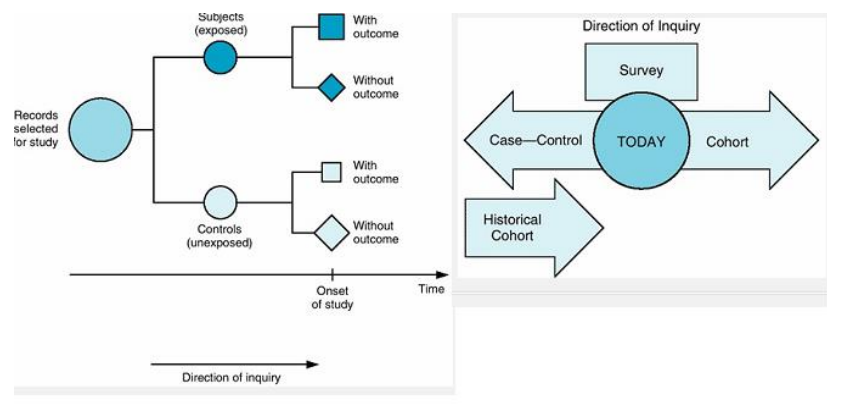

Figure 7: Schematic diagram of a historical cohort and the time relationship among different observational study designs

\section{EXPERIMENTAL STUDIES OR CLINICAL TRIALS}

Experimental studies are generally easier to identify than observational studies in the clinical trial literature. The investigators or Authors of clinical trial organizer reporting experimental studies tend to state explicitly the type of study design used more often than do the reporting observational studies.

Experimental studies in clinical research that involve humans are called clinical trials because their purpose is to draw conclusions about a particular procedure or treatment. Usually clinical trials fall into two categories: those with and those without controls.

Controlled trials are studies in which the experimental drug or procedure is compared with another drug or procedure, sometimes a placebo and sometimes the previously accepted treatment.

Uncontrolled trials are studies in which the investigators' experience with the experimental drug or procedure is described, but the treatment is not compared with another treatment, at least not formally. Because the purpose of an experiment is to determine whether the intervention (treatment) makes a difference, studies with controls are much more likely than those without controls to detect whether the difference is due to the experimental treatment or to some other factor. Thus, controlled studies are viewed as having far greater validity in medicine than uncontrolled studies. The consolidated standard of reporting trials (CONSORT) guidelines reflect an effort to improve the reporting of clinical trials. A comprehensive discussion and illustration of the standard is given by Altman and colleagues (2001)

\section{Randomized Controlled Trials}

Anoneuoid says "Randomized experiments give you unbiased estimates of the causal effect of the treatment that was randomized on the outcome that was measured in whatever population the sample can be considered to be a draw from."

The randomized controlled trial is the epitome of all research designs because it provides the strongest evidence for concluding causation; it provides the best insurance that the result was due to the intervention.

A randomized controlled trial (or randomized control trial; RCT) is a type of scientific (often medical) experiment that aims to reduce certain sources of bias when testing the effectiveness of new treatments; this is accomplished by randomly allocating subjects to two or more groups, treating them differently, and then comparing them with respect to a measured response. One group - the experimental group - has the intervention being assessed, while the otherusually called the control group-has an alternative condition, such as a placebo or no intervention. The groups are followed under conditions of the trial design to see how effective the experimental intervention was. Treatment efficacy is assessed in comparison to the control. There may be more than one treatment group or more than one control group.

The trial may be blinded, in which information which may influence the participants is withheld until after the experiment is complete. A blind can be imposed on any participant of an experiment, including subjects, researchers, technicians, data analysts, and evaluators. Good blinding may reduce or eliminate some sources of experimental bias.

The randomness in the assignment of subjects to groups reduces selection bias and allocation bias, balancing both known and unknown prognostic factors, in the assignment of treatments. Blinding reduces other forms of experimenter and subject biases.

One of the more noteworthy randomized trials is the Physicians' Health Study (Steering Committee of the 
Physicians' Health Study Research Group, 1 989), which investigated the role of aspirin in reducing the risk of cardiovascular disease. One purpose was to learn whether aspirin in low doses reduces the mortality rate from cardiovascular disease. Participants in this clinical trial were over 22,000 healthy male physicians who were randomly assigned to receive aspirin or placebo and were followed over an average period of 60 months.

The investigators found that fewer physicians in the aspirin group experienced a myocardial infarction during the course of the study than did physicians in the group receiving placebo. We discuss several randomized trials as presenting problems. For instance, Borghi and colleagues (2002) compared a traditional low-calcium diet with a diet containing a normal amount of calcium but reduced amount of animal protein and salt for the prevention of recurrent kidney stone formation. The primary outcome was the time to the first recurrence of a symptomatic or presence of a radiographically identified stone. Results indicated that a diet with a normal amount of calcium but reduced animal protein and salt is more effective than the traditional low-calcium diet in reducing the risk of recurrent stones in men with hypercalciuria.

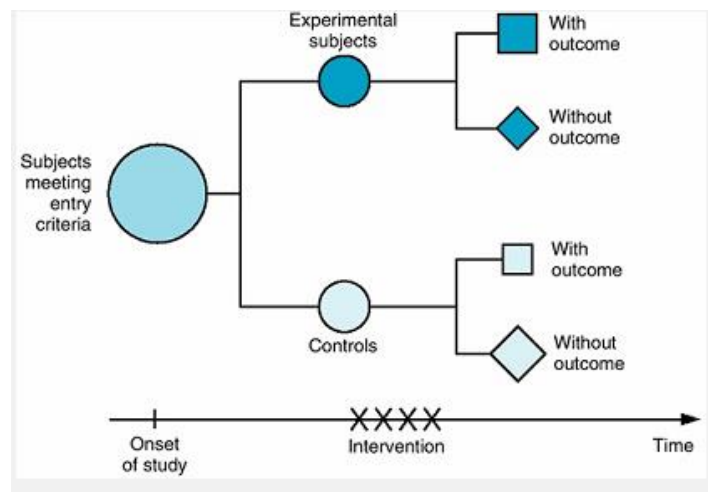

Figure8: Schematic diagram of randomized controlled trial design

\section{Nonrandomized Trials}

Subjects are not always randomized to treatment options. Studies that do not use randomized assignment are generally referred to as nonrandomized trials or simply as clinical trials or comparative studies, with no mention of randomization. $\mathrm{M}$ any investigators believe that studies with nonrandomized controls are open to so many sources of bias that their conclusions are highly questionable. Studies using nonrandomized controls are considered to be much weaker because they do nothing to prevent bias in patient assignment.

For instance, perhaps it is the stronger patients who receive the more aggressive treatment and the higher risk patients who are treated conservatively. An example is a nonrandomized study of the use of a paracervical block to diminish cramping and pain associated with cryosurgery for cervical neoplasia (Harper, 1 997; Chapter 6 presenting problem). This investigator enrolled the first 40 women who met the inclusion criteria in the group treated in the usual manner (no anesthetic block before cryosurgery) and enrolled the next 45 women in the group receiving the paracervical block. This design is not as subject to bias as a study in which patients are treated without regard to any plan; however, it does not qualify as a randomized study and does present some potential problems in interpretation. Whenever patients are assigned to treatments within big blocks of time, there is always the possibility that an important event occurred between the two time periods, such as a change in the method used for cryotherapy. Although that may not have been true in this study, a randomized design would have been more persuasive.

\section{Trials with Self-Controls and Trials with External Controls}

A moderate level of control can be obtained by using the same group of subjects for both experimental and control options. The study by Sauter and colleagues (2002) involved patients who underwent cholecystectomy. Follow-up occurred 1 and 3 months after cholecystectomy to detect changes such as abdominal pain, flatulence, and dyspepsia. This type of study uses patients as their own controls and is called a self-controlled study.

Studies with self-controls and no other control group are still vulnerable to the well-known Hawthorne effect, described by Roethlisberger and colleagues (1 946), in which people change their behavior and sometimes improve simply because they receive special attention by being in a study and not because of the study intervention. These studies are similar to cohort studies except for the intervention or treatment that is involved.

The self-controlled study design can be modified to provide a combination of concurrent and selfcontrols. This design uses two groups of patients: One group is assigned to the experimental treatment, and the second group is assigned to the placebo or control treatment (Figure 2-7). After a time, the 
experimental treatment and placebo are withdrawn from both groups for a "washout" period. During the washout period, the patients generally receive no treatment. The groups are then given the alternative treatment; that is, the first group now receives the placebo, and the second group receives the experimental treatment. This design, called a crossover study, is powerful when used appropriately The third method for controlling experiments is to use controls external to the study. Sometimes, the result of another investigator's research is used as a comparison. On other occasions, the controls are patients the investigator has previously treated in another manner, called historical controls. The study design is illustrated in Figure 2-8.

Historical controls are frequently used to study diseases for which cures do not yet exist and are used in oncology studies, although oncologic studies use concurrent controls when possible. In studies involving historical controls, researchers should evaluate whether other factors may have changed since the time the historical controls were treated; if so, any differences may be due to these other factors and not to the treatment.

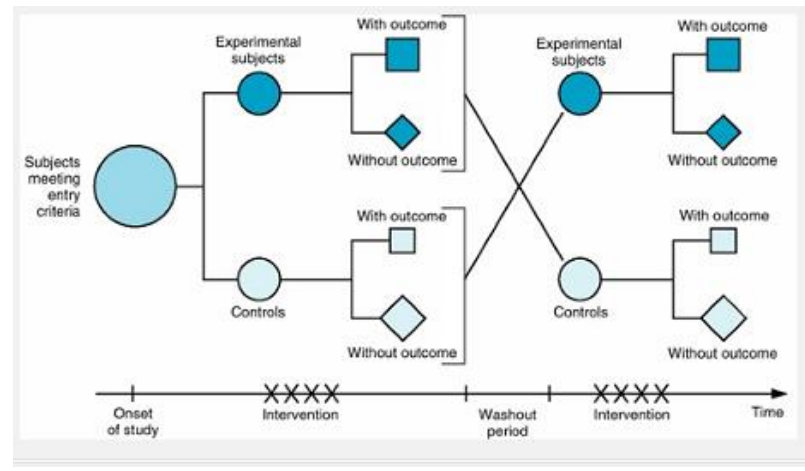

Figure 9: Schematic diagram of trial with crossover
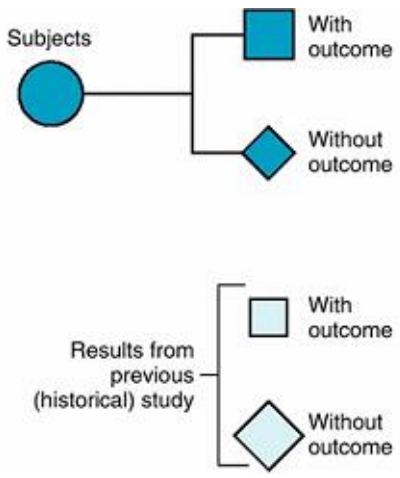

Figure 10: Schematic diagram of trial with external controls

\section{Uncontrolled Studies}

Not all studies involving interventions have controls, and by strict definition they are not really experiments or trials. For example, Crook and associates (1 997) (a presenting problem in Chapter 9) reported the results of a trial of radiotherapy for prostate carcinoma in which patients were followed for at least 12 and for as long as 70 months. The investigators wanted to determine the length of time a patient had no recurrence of the tumor as well as how long the patients survived. They found some differences in the probability of long-term survival in patients who had different tumor classification scores (scores that measure the severity of the tumor). This study was an uncontrolled study because there were no comparisons with patients treated in another manner.

Uncontrolled studies are more likely to be used when the comparison involves a procedure than when it involves a drug. The major shortcoming of such studies is that investigators assume that the procedure used and described is the best one. The history of medicine is filled with examples in which one particular

treatment is recommended and then discontinued after a controlled clinical trial is undertaken. One significant problem with uncontrolled trials is that unproved procedures and therapies can become established, making it very difficult for researchers to undertake subsequent controlled studies. Another problem is finding a significant difference when it may be unfounded. Guyatt and colleagues (2000) identified 13 randomized trials and 17 observational studies in adolescent pregnancy prevention. Six of eight outcomes they examined showed a significant intervention effect in the observational studies, whereas the randomized studies showed no benefit.

\section{META-ANALYSIS \& REVIEW PAPERS}

A type of study that does not fit specifically in either category of observation studies or experiments is called meta-analysis. $M$ eta-analysis uses published information from other studies and combines the results so as to permit an overall conclusion. Metaanalysis is similar to review articles, but additionally includes a quantitative assessment and summary of 
the findings. It is possible to do a meta-analysis of observational studies or experiments; however, a meta-analysis should report the findings for these two types of study designs separately. This method is especially appropriate when the studies that have been reported have small numbers of subjects or come to different conclusions. Veenstra and colleagues (1 999) performed a metaanalysis of infection and central venous catheters. The investigators wanted to know whether catheters impregnated with antiseptic were effective in preventing catheter related bloodstream infection, compared with untreated catheters. They found 12 randomized trials that had addressed this question and combined the results in a statistical manner to reach an overall conclusion about their effectiveness - mainly that the impregnated catheters appear to be effective in reducing the incidence of infection in high-risk patients.

\section{CONCLUSION}

In this article, the aim is to explain the basic designs which are frequently used in clinical trials. This article illustrates the study designs most frequently encountered in the medical literature. In medical research, subjects are observed or experiments are undertaken.

Experiments involving humans are called trials. Experimental studies may also use animals and tissue, although we did not discuss them as a separate category; the comments pertaining to clinical trials are relevant to animal and tissue studies as well. Randomized, controlled clinical trials are the most powerful designs possible in medical research, but they are often expensive and time-consuming. Welldesigned observational studies can provide useful insights on disease causation, even though they do not constitute proof of causes. Cohort studies are best for studying the natural progression of disease or risk factors for disease; case-control studies are much quicker and less expensive. Cross-sectional studies provide a snapshot of a disease or condition at one time, and we must be cautious in inferring disease progression from them. Surveys, if properly done, are useful in obtaining current opinions and practices. Case-series studies should be used only to raise questions for further research.

The discussion pointed out salient features in the design of the presenting problems as we go along, and we will return to the topic of study design again after all the prerequisites for evaluating the quality of journal articles have been presented.

\section{REFERENCES}

1. Rockville, MD. FDA (1997a) Guidance for Industry - the Evaluation of Combination Vaccines for Preventable Diseases: Production, Testing and Clinical Studies. Center for Biologics Evaluation and research, the United States Food and Drug Administration

2. Rockville, MD.FDA (1988) Guideline for the Format and Content of the Clinical and Statistical Sections of New Drug Applications. U.S. Food and Drug Administration.

3. Rockville, MD.FDA (2001) Draft Guidance on the Establishment and Operation of Clinical Trial Data Monitoring Committees, Rockville, MD.

4. Rockville, MD.FDA (1999) Guidance for Industry-Computerized System Used in Clinical Trials. The U.S. Food and Drug Administration.

5. Rockville, MD.FDA (2001) Guidance for Industry on Statistical Approaches to Establishing Bioequivalence. Center for Drug Evaluation and Research, Food and Drug Administration.

6. Rockville, MD FDA (2001) Bioresearch Monitoring-Compliance Program Guidance Manual. The U.S. Food and Drug Administration.

7. Rockville, MD FDA (2003) Draft Guidance on Developing Medical Imaging Drugs and Biological Products, Part 3: Design, Analysis and Interpretation of Clinical Studies. The United States Food and Drug Admin-istration, Rockville, MD.

8. Rockville, MD FDA (2003) Guidance for Industry on Part 11, Electronic Records; Electronic Signatures-Scope and Application. The U.S. Food and drug Administration, Rockville, MD.

9. VICH Steering committee (2000) VICH Guideline GL9 on Good Clinical Practice. EMEA.

10. U.S. National Archives and Records Administration (2012) Code of federal regulations. Title 21. Volume 1, Part 50, Protection of Human Subjects.

11. U.S. National Archives and Records Administration (2012) Code of federal regulations. Title 21. Volume 1, (Part 56, Institutional Review Boards. Part 312, Investigational New Drug Application. Part 314, Applications for FDA Approval to Market a New Drug.)

12. VICH Steering committee (2000) VICH Guideline GL9 on Good Clinical Practice. EMEA.

13. Chow SC, Liu JP (2004) Design and Analysis of Clinical Trials: Concepts and Methodologies. $\left(2^{\text {nd }}\right.$ edn), John Wiley and Sons, New York 
International Journal of Engineering Applied Sciences and Technology, 2020

Vol. 4, Issue 11, ISSN No. 2455-2143, Pages 498-508

Published Online March 2020 in IJEAST (http://www.ijeast.com)

14. Shein-Chung Chow1 and Shih-Ting Chiu(2013) A Note on Design and Analysis of Clinical Trials

15. ICH (1995) International Conference on Harmonization Tripartite Guideline
16. Caiola.E et.al (2000) Factors influencing the selection of general internal medicine Fellowship programs. J GEN INTERN MED 15, 656 